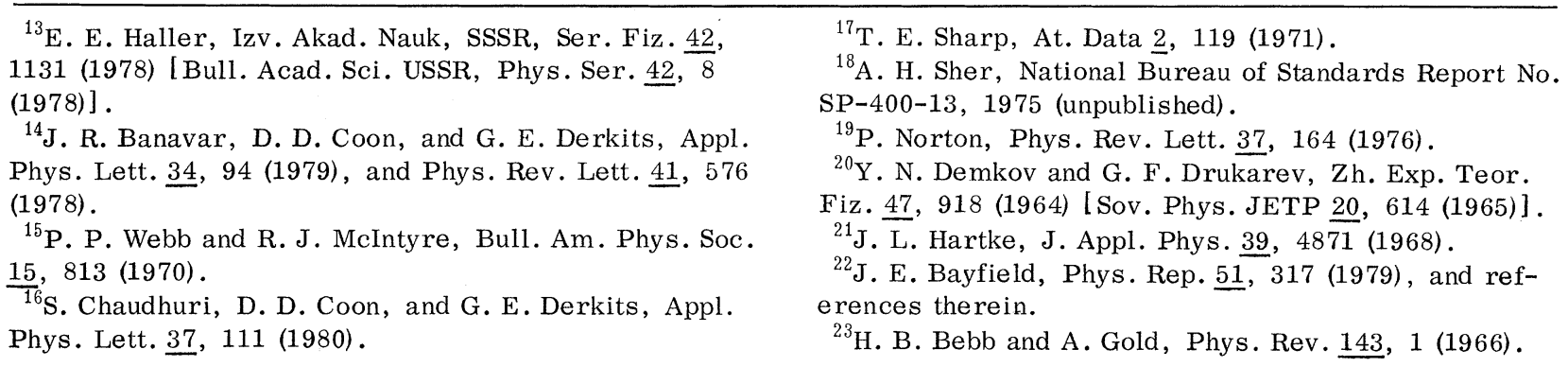

\title{
Predictions of a Theory of Quark Confinement
}

\author{
Gerhard Mack \\ II. Institut für Theoretische Physik der Universität Hamburg, D-2000 Hamburg 36, Germany \\ (Received 31 March 1980)
}

\begin{abstract}
A theory of quark confinement is proposed which uses only the simplest of approximations. It explains persistence of quark confinement in Yang-Mills theories with gauge group SU(2) or SU(3) as a consequence of asymptotic freedom in perturbation theory and of the known phase structure of $\boldsymbol{Z}(2)$ and $\boldsymbol{Z}(3)$ lattice gauge theory. Predictions are derived which can in principle be tested by computer simulation. Some are already tested by results of Creutz. They are in good agreement.

PACS numbers: $12.40 . \mathrm{Bb}, 11.10 . \mathrm{Np}$
\end{abstract}

In this Letter, I present a theory of quark confinement in gauge field theories which uses only the simplest of approximations. It explains quark confinement in theories with gauge groups SU(2) or SU(3) on continuous space-time as a consequence of asymptotic freedom in perturbation theory and of the known phase structure of lattice gauge theories with gauge group $Z(2)$ and $Z(3)$, respectively. It is based on a set of hypotheses, concerning the physical mechanism of quark confinement, which will be formulated below. These hypotheses are motivated by certain rigorous mathematical results on the statistical mechanics of quark confinement. ${ }^{1,2}$ They cannot be rigorously derived themselves at the present time, but I give plausibility arguments. Predictions will be derived that can in principle be tested by Monte Carlo computations for a pure SU(2) Yang-Mills theory on a finite lattice. A test of some of these results is possible with already available data of Creutz. ${ }^{3}$ Very good agreement is observed.

I shall use the formalism of Euclidean quantum field theory to formulate the present theory in the language of classical statistical mechanics. To be specific, I consider a pure Yang-Mills theory with gauge group SU(2) with coupling parameter $\beta=4 / g_{0}{ }^{2}$ on a lattice of (arbitrarily small) lattice spacing $a$. This theory will be compared with a lattice gauge theory with gauge group $Z(2)$ and coupling parameter $\beta_{\text {eff }}(d)$ on a lattice of larger spacing $d$. Only certain general properties of $\beta_{\text {ef } f}(d)$ will be essential for the working of the theory. They will be described below.

The basic hypotheses of this theory are as follows:

(1) Quark confinement is due to a vortex condensation mechanism. ${ }^{2,4}$

(2) It makes some sense to talk about a metastable phase [of the SU(2) theory] without quark confinement and vortex condensation which can be treated by perturbation theory. ${ }^{1}$

(3) Vortices of thickness $d$ condense, if this metastable phase is unstable against their condensation.

(4) Such instability occurs if

$$
\beta_{\text {eff }}^{\text {meta }}(d) \leqslant \beta_{c} \text {. }
$$

The superscript "meta" indicates that $\beta_{\text {eff }}(d)$ is to be computed for the metastable phase; that is, by perturbation theory. $\beta_{c} \approx 0.44$ is the inverse transition temperature of the $Z(2)$ theory.

(5) Condensation of vortices of thickness $d$ leads to suppression factors in the Wilson loop expectation value, etc., which are the same as in the $Z(2)$ theory with lattice spacing $d$ and coupling parameter $\beta_{\text {eff }}(d)$.

The random variables of our lattice gauge theories are gauge fields $U(b)$ in $\mathrm{SU}(2)$ and $\sigma(b)= \pm 1$ 
that are attached to links $b$ of the lattices. Our normalization conventions for the coupling parameters are the standard ones. Thus, the action for the SU(2) theory and the $Z(2)$ theory with coupling parameter $\beta$ is ${ }^{5}$

$$
L(U)=\frac{1}{2} \beta \sum_{p} \operatorname{tr}[U(\dot{p})-1]
$$

and

$$
L(\sigma)=\beta \sum_{p}[\sigma(\dot{p})-1],
$$

respectively. Here, $U(\dot{p})=U\left(b_{4}\right) \cdots U\left(b_{1}\right)$ and $\sigma(\dot{p})$ are the parallel transporters around a plaquette $p$ with boundary consisting of links $b_{1}, \cdots, b_{4}$. Summation is over all unoriented plaquettes of the lattice.

We shall now turn to a brief discussion of arguments for the validity of the above hypotheses.

Hypotheses (1) and (2) have been discussed in the literature before..$^{1,2,4}$ Hypothesis (3) is selfexplanatory. To arrive at hypothesis (4) we appeal to a generalization of Fisher's microdomainwall theory of ferromagnets. ${ }^{6}$ Vortices are world sheets of closed loops of magnetic flux. They are the analog of domain walls in ferromagnets. ${ }^{1,2,4}$ Consider a vortex of thickness $d$ and divide it into $N$ blocks of size $d \times d \times d \times d$. Its chemical potential

$$
\mu=E-S / \beta \text {. }
$$

Here, $S$ equals the logarithm of the number of configurations of $N$ blocks that form a vortex container (closed two-dimensional sheet of thickness $d)^{1,2}$ and $E$ equals the energy in case of a vortex without internal structure, resp. the internal free energy which is obtained from the energy by subtracting $\beta^{-1}$ times the entropy associated with the fluctuations in the internal structure of the vortex. We set

$$
\beta_{\text {eff }}(d)=\beta E / 2 N \text {. }
$$

The entropy $S$ is the same as in the standard $Z(2)$ theory. The chemical potential $\mu$ will depend on the thickness $d$. Vortices of thickness $d$ will condense if $\mu / N \leqslant 0$. By comparing with the analogous argument for a $Z(2)$ theor ${ }^{7}$ we see that vortices of thickness $d$ will condense in the SU(2) theory if and only if (thin) ${ }^{1}$ vortices condense in the $Z(2)$ theory with coupling parameter $\beta_{\text {eff }}(d)$. But it is known that the $Z(2)$ theory has two phas$\mathrm{es}^{8}$ which are separated by a first-order phase transition $^{9}$ at $\beta=\beta_{c}=0.44$ : a high-temperature phase with confinement and vortex condensation $\beta \leqslant \beta_{c}$, and a low-temperature phase at $\beta \geqslant \beta_{c}$ with- out them. Note that the interaction between vortices is not simply ignored in this approach. It affects the chemical potential and is thus taken into account in a kind of mean-field approximation. Mean-field approximation is an effective way of dealing with possible long-range interactions.

Hypothesis (5) is a corollary of the philosophy of a $Z(2)$ theory of quark confinement as it was discussed in Ref. 1. One could also try to justify hypothesis (5) by appeal to considerations of conditional expectation values, generalizing considerations of Refs. 1 and 2 , but I do not intend to discuss this here.

In agreement with hypothesis (2) we will assume that the two leading nonvanishing terms in the perturbation expansion of $\beta_{\text {eff }}^{\text {met a }}(d)$ give an accurate approximation for sufficiently large $d$ and any bare coupling constant $g_{0}$ which is such that $\beta_{\text {eff }}^{\text {met a }}(d) \geqslant \beta_{c}$. We assume that $\beta_{\text {eff }}(d)$ possesses a continuum limit in perturbation theory. Its perturbation series will then be expressible in terms of the perturbation-theoretic renormalizationgroup invariant $x$. Because of the explicit factor $\beta$ in Eq. (5) it starts with a (possibly vanishing) term proportional to $\beta$ and has therefore the following form:

$$
\begin{aligned}
\beta_{\text {eff }}^{\operatorname{met}}(d) & =a_{-1} x+a_{0}+a_{1} / x+\cdots, \\
x & =\beta-\left(11 / 6 \pi^{2}\right) \ln \left(d^{2} / a^{2}\right)+x_{0}+\cdots .
\end{aligned}
$$

It is expected that $\beta_{\text {ef }}(d)$ increases monotonically with $\beta$, and reaches values larger than $\beta_{c}$ for sufficiently large $\beta$ and fixed $d$. This imposes some constraints on the coefficients $a_{k}$ in its perturbation expansion. $x_{0}$ may be chosen by convention, for instance, so that a term $a_{2} / x^{2}$ in expansion (6) is absent. The - sign in the expression of $x$ reflects asymptotic freedom. ${ }^{10}$

We will now show that confinement of static quarks in the SU(2) theory will persist for arbitrarily small values of the bare coupling constant $g_{0}$, and in the continuum limit, if our hypotheses are valid.

According to hypotheses (3) and (4), vortices of some thickness $d_{c}=d_{c}(\beta)$ will condense if the following equation has a finite solution $d_{c}$ :

$$
\beta_{\mathrm{eff}}^{\mathrm{meta}}\left(d_{c}\right)=\beta_{c} \text {. }
$$

We see from Eq. (6) that Eq. (7) always has a finite solution $d_{c}$, even for arbitrarily large values of $\beta=4 / g_{0}^{2}$. It is given by

$$
d_{c}{ }^{2}=a^{2} \exp \left[\left(6 \pi^{2} / 11\right)\left(\beta-\beta_{1}\right)\right]
$$


with a value of $\beta_{1}$ that is determined by the coefficients in the perturbation expansion (6). According to hypothesis (5) the string tension in the SU(2) theory is then

$$
\alpha=\alpha_{Z(2)}\left(\beta_{c}-\epsilon\right) a^{2} / d_{c}{ }^{2} .
$$

This equation involves the string tension in the $Z(2)$ theory just above the temperature $\beta_{c}{ }^{-1}$. Putting everything together we obtain the final result for the string tension:

$$
\alpha=\alpha_{0} \exp \left[-\left(6 \pi^{2} / 11\right)\left(\beta-\beta_{1}\right)\right]
$$

if $d_{c}(\beta)>a$, i.e., $\beta>\beta_{1}$, with

$$
\alpha_{0}=\alpha_{Z(2)}\left(\beta_{c}-\epsilon\right) \approx 0.6 a^{-2} \text {. }
$$

The last estimate of the constant $\alpha_{0}$ is obtained by appeal to high-temperature expansions for the $Z(2)$ theory. ${ }^{11}$ Since the phase transition of the $Z$ (2) model is of first order, this is not an unreasonable approximation.

We say that the theory is in the high-temperature phase if arbitrarily thin vortices are condensed. It begins at the value $\beta=\beta_{1}$ where $d_{c} / a$ $=1$; this signals the disappearance of the nonempty range of distances $a<d<d_{c}$ where perturbation theory applies. Relation (10) is only valid outside the high-temperature phase. In the hightemperature phase we set $d_{c}=a$. The string tension can be computed by high-temperature expansions in this case $\mathrm{e}^{5,12}$

$$
\alpha=-a^{-2} \ln (\beta / 4)+\cdots, \text { for } \beta \leqslant \beta_{1} .
$$

The parameter $\beta_{1}$ can be computed from the relation $\alpha\left(\beta_{1}\right)=\alpha_{0}$ by using the high-temperature expansions for $\alpha$ and $\alpha_{0}$. This gives $\beta_{1} \approx 2.0$. We are thus able to compute the string tension without any free parameter. (See Fig. 1.)

We see that the string tension is finite for all values of $g_{0}$. Let us turn to the continuum limit $a \rightarrow 0$. We may regard the string tension $\alpha$ as a kind of renormalized coupling constant, and hold it fixed in the continuum limit. According to Eqs. (10) and (11) this requires that the bare coupling constant behave as

$$
g_{0}{ }^{2} \sim-\left(12 \pi^{2} / 11\right)(\ln \rho a)^{-1} \text { as } a \rightarrow 0,
$$

with $\rho$ some constant mass. Such behavior of the bare coupling constant is in agreement with the hypothesis of asymptotic freedom in the physical theory.

According to Creutz, ${ }^{3}$ expression (10) gives a very good fit to Monte Carlo data for the string tension, for a value of $\beta_{1}=2.09$. The biggest values of $\beta$ where data are available correspond to

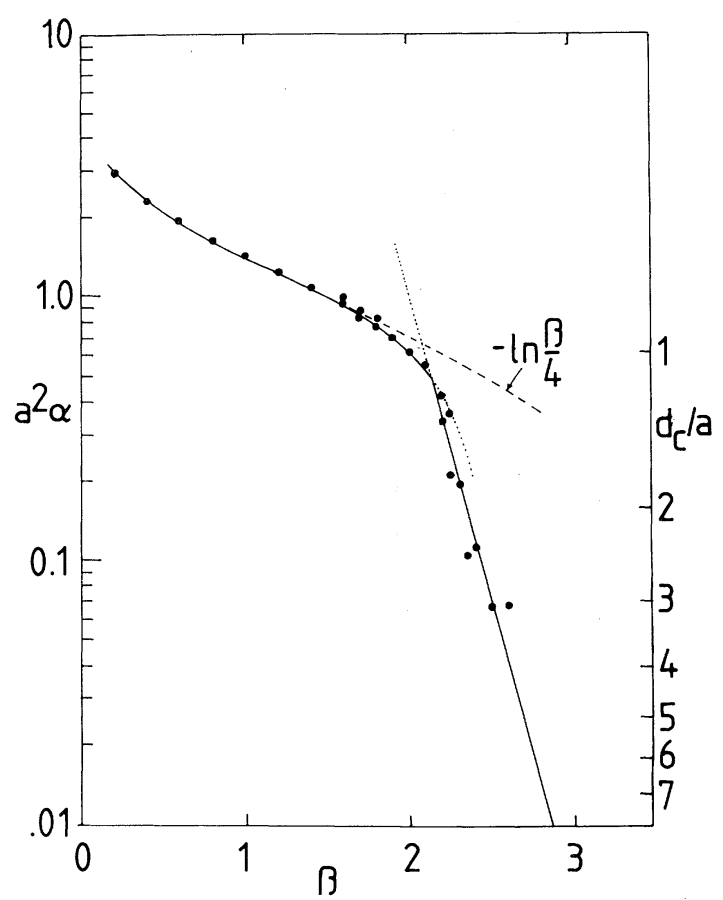

FIG. 1. The string tension $\alpha$ and corresponding values of $d_{c}$. The black dots represent the Monte Carlo data of Creutz (Ref. 3). The black lines are a fit to Eq. (10) with $\beta=2.09$ (large $\beta$ ) and the prediction of high-temperature cluster expansions of Münster (Ref. 12) up to tenth order (low $\beta$ ).

$d_{c} / a$ between 3 and 4 .

In order to make some further predictions we consider the following quantity which was introduced by 't Hooft.

$$
\nu(d)=-\frac{1}{2}\left(\frac{d}{d^{\prime}}\right)^{2}\left(\ln \frac{Z \text { (block,t.p.b.c. })}{Z \text { (block, p.b.c. })}\right)
$$

$Z(\cdots)$ are partition functions in the $\mathrm{SU}(2)$ theory of a block of size $d \times d \times d^{\prime} \times d^{\prime}\left(d^{\prime} \geqslant d\right)$ with periodic boundary conditions (p.b.c.) and twisted periodic boundary conditions (t.p.b.c.). They are obtained from periodic ones by a singular gauge transformation. ${ }^{1,2,4}$ The singular gauge transformation forces a quantum of magnetic flux in a particular direction into the block. It agrees with an ordinary gauge transformation in a neighborhood of every point of the boundary of the block, but it changes the parallel transporter $U(C)$ into $-U(C)$ for every closed path $C$ on the boundary of the block which winds once around the intersection of the $x^{3}-x^{4}$ plane with the block. For $d^{\prime}$ large, $\nu(d)$ should be independent of $d^{\prime}$. 


\section{High-temperature expansions predict that}

$$
\nu(d)=(d / a)^{2} \exp \left(-\alpha d^{2}\right) \text { for large } d / a,
$$

where $\alpha$ is the string tension. This is true both in the $Z(2)$ theory and in the SU(2) theory. ${ }^{12}$ To get a result for the $\mathrm{SU}(2)$ theory which is valid outside the high-temperature phase, we identify it again with a $Z(2)$ theory just above $\beta_{c}$, and I use Eq. (15) for this theory. The lattice spacing is then $d_{c}$ and the string tension $\alpha_{0}$. Inserting Eq. (9), we get

$$
\nu(d)=\left[d / d_{c}(\beta)\right]^{2} \exp \left(-\alpha d^{2}\right)
$$

outside the high-temperature phase. This is supposed to be valid for $d$ substantially larger than $d_{c}$. Hypothesis (5) alone predicts that (16) should be true except possibly for a slowly varying proportionality factor. This relation could be tested by Monte Carlo computations. ${ }^{13}$

It is also of some interest to investigate whether $\nu(d)$ can be used as an approximation to the effective $Z(2)$ coupling constant,

$$
\beta_{\text {eff }}(d)=\nu(d) 。
$$

To obtain this approximation, one imagines cutting a piece of area $d^{\prime 2}$ out of a vortex sheet of thickness $d$ and simulating the effect of its environment by imposing periodic boundary conditions. Unfortunately this mutilates the effect of the interaction of vortices: Their interaction is simulated by the interaction of the blocks with their periodically repeated selves through their own boundaries. The residual interaction could lead to a shift of $\beta_{c}$. The appropriate value of $\beta_{c}$ can be found from the requirement that the two independent ways of determining $\beta_{1}$ (from high-temperature expansions and from the perturbation series - see above) yield the same result.

In any case, $\nu(d)$ should behave very similarly to $\beta_{\text {eff }}(d)$, and one could therefore test our assumptions concerning the range of validity of low-order perturbation theory through Monte Carlo computations ${ }^{13}$ of $\nu(d)$. The two leading nonvanishing orders of its perturbation expansion should give accurate results for all values of $\beta$ where $d \leqslant d_{c}(\beta)$. [The coefficients in the expansion could be determined by computations for large $\beta$ (and fixed $d$ ) where the saddle-point method is surely applica- ble.] Deviations might result from instanton effects. ${ }^{14}$

The quantity $\nu$ can also be defined for theories with matter fields (e.g. quark fields). In this case, only the gauge fields need satisfy periodic and twisted periodic boundary conditions, whereas free boundary conditions are adopted for the matter fields.

Let me finally point out that in the present theory persistence of quark confinement is not tied to the absence of a phase transition. Validity of these hypotheses is supposed to be model independent, and the modified SU(2) model of Petkova and the author, ${ }^{2}$ which is known to have a phase transition at some $\beta>\beta_{c}$, is predicted to behave in the same way as discussed here for large $\beta$.

The author is indebted to E. Pietarinen, G. Münster, and K. Symanzik for discussions.

\footnotetext{
${ }^{1}$ G. Mack, in “Recent Developments in Gauge Theories," edited by G. 't Hooft et al. (Plenum, New York, to be published).

${ }^{2}$ G. Mack and V. B. Petkova, Ann. Phys. (N. Y.) $\underline{123}$, 447 (1979), and $\underline{125}, 117$ (1980).

${ }^{3}$ M. Creutz, Phys. Rev. D 21, 2309 (1980).

${ }^{4}$ G. 't Hooft, Nucl. Phys. B153, 141 (1979).

${ }^{5}$ K. G. Wilson, Phys. Rev. D 10 , 2445 (1974).

${ }^{6}$ M. E. Fisher, J. Appl. Phys. 38 , 981 (1967).

${ }^{7}$ T. Yoneya, Nucl. Phys. B144, 195 (1978).

${ }^{8}$ F. J. Wegner, J. Math. Phys. 12, 2259 (1971); B. Balian, J. M. Drouffe, and C. Itzykson, Phys. Rev. D 10,3376 (1974), and $\underline{11}, 2098$ (1975).

${ }^{9} \mathrm{M}$. Creutz, Phys. Rev. Lett. $\underline{43}, 553$ (1979).

${ }^{10}$ D. J. Gross and F. Wilzcek, Phys. Rev. Lett. $\underline{30}$, 1343 (1973), and Phys. Rev. D 8 , 3633 (1973); H. D. Politzer, Phys. Rev. Lett. 30, 1346 (1973).

${ }^{11}$ N. Kimura, "Critical Properties of $Z(2)$ Lattice Gauge Systems from Strong Coupling Expansions" (to be published).

${ }^{12}$ G. Münster, Phys. Lett. 95B, 59 (1980), and Ph.D. thesis, Universität Hamburg, 1980, DESY Report No. DESY-80/44 (unpublished).

${ }^{13}$ After completion of this work, some Monte Carlo data for twisted boundary conditions are now available: J. Groenveld, J. Jurkiewicz, and C. P. Korthals Altes, Phys. Lett. 92B, 312 (1980); G. Mack and E. Pietarinen, Phys. Lett. 94B, 397 (1980).

${ }^{14}$ C. G. Callan, R. F. Dashen, and D. J. Gross, Phys. Rev. Lett. $\underline{44}, 435$ (1980), and Phys. Rev. D $\underline{20}, 3279$ (1979) .
} 